\title{
Quadratic Invariance is Necessary and Sufficient for Convexity
}

\author{
Laurent Lessard ${ }^{1} \quad$ Sanjay Lall ${ }^{2}$ \\ American Control Conference, pp. 5360-5362, 2011
}

\begin{abstract}
In decentralized control problems, a standard approach is to specify the set of allowable decentralized controllers as a closed subspace of linear operators. This then induces a corresponding set of of Youla parameters. Previous work has shown that quadratic invariance of the controller set implies that the the set of Youla parameters is convex. In this short note, we prove the converse. We thereby show that the only decentralized control problems for which the set of Youla parameters is convex are those which are quadratically invariant.
\end{abstract}

\section{Introduction}

In this paper, we consider the feedback control of linear plants subject to structural information constraints on the set of admissible controllers. In particular, we are interested in knowing when the optimal controller is linear, and when finding it can be reduced to a convex optimization problem. In general, such decentralized controlller synthesis problems are hard [1], and even when the noise is Gaussian and the cost function is quadratic, the optimal controller may not be linear. Furthermore, finding the optimal linear controller amounts to solving a nonconvex optimization problem [7].

Despite this difficulty, some problems in this class are tractable, and much work has been done in recent years to characterize them. See for example [5] and references therein. In recent work $[5,3]$, the notion of quadratic invariance is developed. This condition guarantees that the optimal controller will be linear, and, when the quadratic invariance condition holds, finding the optimal controller amounts to solving a convex optimization problem.

This result holds in great generality. For example, in the matrix case, suppose $G \in \mathbb{R}^{m \times n}$ and $S \subset \mathbb{R}^{n \times m}$ is a subspace, and define the set

$$
h(S)=\left\{-K(I-G K)^{-1} \mid K \in S\right\}
$$

\footnotetext{
${ }^{1}$ L. Lessard is with the Department of Aeronautics and Astronautics at Stanford University, Stanford, CA 94305, USA. lessard@stanford.edu

${ }^{2} \mathrm{~S}$. Lall is with the Department of Electrical Engineering and Aeronautics and Astronautics at Stanford University, Stanford, CA 94305, USA. lall@stanford.edu

${ }^{3}$ This work was supported by AFOSR MURI project titled MultiLayer and Multi-Resolution Networks of Interacting Agents in Adversarial Environments
}

Suppose that $I-G K$ is invertible for all $K \in S$, so that $h(S)$ is well-defined. The idea, in the setting of more general linear operators, is that $S$ is the set of controllers with the desired decentralization structure, and $h(S)$ is the set of corresponding Youla parameters.

The set $S$ is called quadratically invariant if

$$
K G K \in S \text { for all } K \in S
$$

Previous results have shown that if $S$ is quadratically invariant, then $h(S)=S$, and the converse of this result is also true. The practical importance of this result is that if the set of controllers $S$ is quadratically invariant, then the corresponding set of Youla parameters is convex.

Notice however that this result does not preclude the possibility that $h(S)$ is convex, but not equal to $S$. The convexity of $h(S)$ is of tremendous importance in decentralized control; if $h(S)$ is convex, then one may find the optimal controller via convex optimization.

Main result. The question thus arises whether, given the system $G$, there is any non-quadratically-invariant set $S$ for which $h(S)$ is convex. Informally, such sets would correspond to convex decentralized control problems which are not quadratically invariant.

In this paper we show that the answer is negative. We show that if $h(S)$ is convex, then $S$ must be quadratically invariant. This therefore implies that quadratic invariance is necessary and sufficient for the set $h(S)$ to be a convex set. We prove the result in a general setting where $G$ and $K$ are bounded linear operators on Banach spaces.

\subsection{Preliminaries}

If $\mathcal{X}$ and $\mathcal{Y}$ are Banach spaces, we denote by $\mathcal{L}(\mathcal{X}, \mathcal{Y})$ the set of all bounded linear operators $A: \mathcal{X} \rightarrow \mathcal{Y}$. We abbreviate $\mathcal{L}(\mathcal{X}, \mathcal{X})$ to $\mathcal{L}(\mathcal{X})$. A map $A \in \mathcal{L}(\mathcal{X})$ is called invertible if there exists $B \in \mathcal{L}(\mathcal{X})$ such that $A B=B A=I$. Define the resolvent set $\rho(A)=$ $\{\lambda \in \mathbb{C} \mid(\lambda I-A)$ is invertible $\}$. This set is always open, and possibly disconnected, though it contains all sufficiently large $\lambda \in \mathbb{C}$. We will denote by $\rho_{u c}(A)$ the unbounded connected component of $\rho(A)$.

In this paper, we will use the same framework as in [4]. Suppose $\mathcal{U}, \mathcal{W}, \mathcal{Y}, \mathcal{Z}$ are Banach spaces over $\mathbb{R}$. 
We can think of these spaces as the controlled inputs, disturbances, measurements, and regulated outputs, respectively. Suppose we also have the plant $P \in$ $\mathcal{L}(\mathcal{W} \times \mathcal{U}, \mathcal{Z} \times \mathcal{Y})$ and controller $K \in \mathcal{L}(\mathcal{Y}, \mathcal{U})$. We partition $P$ in the obvious way, and connect the controller to the plant as in Figure 1. For the remainder of this paper, we will use $G=P_{22}$ as an abbreviation.

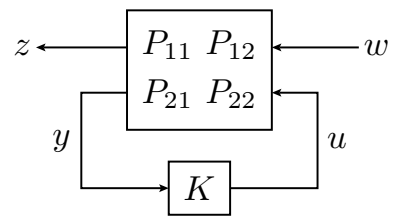

Figure 1: Closed-loop interconnection between a plant $P$ and controller $K$

The resulting closed-loop map (from $w$ to $z$ ) is given by the linear fractional transform $f(P, K) \in \mathcal{L}(\mathcal{W}, \mathcal{Z})$, where

$$
f(P, K)=P_{11}+P_{12} K(I-G K)^{-1} P_{21}
$$

This interconnection is well-posed whenever $I-G K$ is invertible. More formally, we may define the set of admissible controllers $M \subset \mathcal{L}(\mathcal{Y}, \mathcal{U})$ as:

$$
M=\{K \in \mathcal{L}(\mathcal{Y}, \mathcal{U}) \mid(I-G K) \text { is invertible }\}
$$

Here, we only consider controllers which are bounded linear operators. Define the set $N \subset M$, which we will need later

$$
N=\left\{K \in \mathcal{L}(\mathcal{Y}, \mathcal{U}) \mid 1 \in \rho_{u c}(G K)\right\}
$$

\subsection{Optimization}

It is convenient to define the function $h_{G}: M \rightarrow M$

$$
h_{G}(K)=-K(I-G K)^{-1}
$$

We will often omit the subscript $G$ when it is clear by context. Note that $h$ is an involution. That is, $h$ is its own inverse: $h(h(K))=K$ for all $K \in M$. It follows that $h$ is a bijection from $M$ to $M$.

Our goal is to solve the optimization problem

$$
\begin{aligned}
\operatorname{minimize} & \left\|P_{11}+P_{12} K(I-G K)^{-1} P_{21}\right\| \\
\text { subject to } & K \in S \cap M
\end{aligned}
$$

Where $S \subset \mathcal{L}(\mathcal{Y}, \mathcal{U})$ is a closed subspace. Define the Youla parameter $Q=h(K)$ and use the involution property of $h$ to rewrite (1) as

$$
\begin{aligned}
\operatorname{minimize} & \left\|P_{11}-P_{12} Q P_{21}\right\| \\
\text { subject to } & Q \in h(S \cap M)
\end{aligned}
$$

Formulation (1) optimizes a potentially nonconvex function; though the constraint set $S$ is a subspace. Viewed this way, the complexity of the optimization arises because of the non-convexity of the objective function.
Formulation (2) optimizes a convex function, but the feasible set could be nonconvex. Using this change of variables, the objective function is now convex, but there is a non-convex constraint. In the next section, we define quadratic invariance, a property that ensures that the set $h(S \cap M)$ is convex.

\subsection{Quadratic Invariance}

Definition 1. The subspace $S \subset \mathcal{L}(\mathcal{Y}, \mathcal{U})$ is said to be quadratically invariant with respect to $G$ if $K G K \in S$ for all $K \in S$.

Theorem 2 (from [4]). Suppose that $G \in \mathcal{L}(\mathcal{U}, \mathcal{Y})$, and $S \subset \mathcal{L}(\mathcal{Y}, \mathcal{U})$ is a closed subspace. Further suppose that $N \cap S=M \cap S$. Then $S$ is quadratically invariant with respect to $G$ if and only if $h(S \cap M)=S \cap M$.

So if $S$ is quadratically invariant with respect to $G$, the optimization problem (2) is equivalent to

$$
\begin{aligned}
\operatorname{minimize} & \left\|P_{11}-P_{12} Q P_{21}\right\| \\
\text { subject to } & Q \in S \cap M
\end{aligned}
$$

If $Q^{*}$ solves this problem, then the $K^{*}$ that solves (1) is found via $K^{*}=h\left(Q^{*}\right)$. In most practical problems of interest, well-posedness requirements force the optimal $Q$ to lie within $M$, and so we may find it by solving the convex optimization problem

$$
\begin{aligned}
\operatorname{minimize} & \left\|P_{11}-P_{12} Q P_{21}\right\| \\
\text { subject to } & Q \in S
\end{aligned}
$$

\section{Main Result}

The original motivation was to find conditions which ensure convexity of the set $\left\{P_{11}-P_{12} h(K) P_{21} \mid K \in S\right\}$, assuming $h(K)$ is well-defined for all $K \in S$. Quadratic invariance achieves this by finding a necessary and sufficient condition under which $h(S)=S$. But in principle, one could also achieve convexity if $h(S)$ is any convex set. In this section, we show that this never occurs. If $h(S)=T$ where $T$ is convex, then $T=S$.

Definition 3. Suppose $\mathcal{X}$ is a Banach space over $\mathbb{R}$, and $S \subset \mathcal{X}$. We call $S$ a double-cone if for all $x \in S$ and $\alpha \in \mathbb{R}$, we have $\alpha x \in S$.

Note that every subspace is a double-cone, but not all double-cones are subspaces.

Definition 4. Suppose $\mathcal{X}$ is a Banach space over $\mathbb{R}$, and $T \subset \mathcal{X} . \quad W e$ call $T$ a star-set if for all $x \in T$ and $\alpha \in[0,1]$, we have $\alpha x \in T$.

Note that every convex set is a star-set, but not all starsets are convex.

We now present our main result: $h(S \cap M)$ is either nonconvex, or equal to $S \cap M$. 
Theorem 5. Suppose $S \subset \mathcal{L}(\mathcal{Y}, \mathcal{U})$ is a closed doublecone, $T \subset \mathcal{L}(\mathcal{Y}, \mathcal{U})$ is a star-set, and $h(S \cap M)=T \cap M$, then $T \cap M=S \cap M$.

Proof. Fix some $K \in S \cap M$. Since $K \in M, I-G K$ is invertible, and $1 \in \rho(G K)$. The resolvent set of a bounded linear operator is an open set, so there exists a sufficiently small $\epsilon>0$ such that $1-\alpha \in \rho(G K)$ for all $0 \leq \alpha<\varepsilon$. For any such $\alpha$, it follows that $I-(1-\alpha) G K$ is invertible. It follows that $(I-(1-\alpha) G K)(I-G K)^{-1}$ is invertible as well. Expanding this expression, we find that it is equal to $I-\alpha G h(K)$. Thus $\alpha h(K) \in M$.

Also, $K \in S, h(K) \in T$, and so $\alpha h(K) \in T$ whenever $0 \leq \alpha \leq 1$, because $T$ is a star-set. It follows that for $\alpha \in[0, \varepsilon), \alpha h(K) \in T \cap M$.

Now apply $h$ to both sides: $h(\alpha h(K)) \in h(T \cap M)=$ $S \cap M$, where we made use of the involutive property of $h$. Expanding $h(\alpha h(K))$, we find that it is equal to $\alpha K(I-(1-\alpha) G K)^{-1}$. Since $S$ is a double-cone, we may multiply this expression by $-1 / \alpha$, and the result will still lie in $S$. Thus, $-K(I-(1-\alpha) G K)^{-1} \in S$. Now define the function $g:[0, \varepsilon) \rightarrow \mathcal{L}(\mathcal{Y}, \mathcal{U})$ by

$$
g(\alpha)=-K(I-(1-\alpha) G K)^{-1} .
$$

Since $S$ is closed, and $g(\alpha) \in S$ for $\alpha \in[0, \varepsilon)$, then

$$
\lim _{\alpha \rightarrow 0^{+}} g(\alpha) \in S .
$$

Since $(I-G K)$ is invertible, $g$ is right-continuous at 0 . So we may take the limit $\alpha \rightarrow 0^{+}$by simply evaluating $g$ at $\alpha=0$. Thus, we conclude that $h(K) \in S$. Now $h$ is a bijection from $M$ to $M$, and so we actually have $h(K) \in S \cap M$. Since $K$ was an arbitrary element of $S \cap M$, it follows that $h(S \cap M) \subset S \cap M$. Using the involutive property of $h$ once more, $h(S \cap M)=S \cap M$, as required.

Corollary 6. Suppose $S \subset \mathcal{L}(\mathcal{Y}, \mathcal{U})$ is a closed subspace, $T \subset \mathcal{L}(\mathcal{Y}, \mathcal{U})$ is convex, and $h(S \cap M)=T \cap M$. Then $h(S \cap M)=S \cap M$.

Proof. This follows from Theorem 5, since all subspaces are double-cones, and all convex sets are star-sets.

Corollary 7. Suppose $G \in \mathcal{L}(\mathcal{U}, \mathcal{Y})$ and $S \subset \mathcal{L}(\mathcal{Y}, \mathcal{U})$ is a closed subspace such that $I-G K$ is invertible for all $K \in S$, and $M=N$. Then the set

$$
\left\{K(I-G K)^{-1} \mid K \in S\right\}
$$

is convex if and only if $S$ is quadratically invariant with respect to $G$.

Proof. Note that $N \cap S=M \cap S=S$ in this case. Sufficiency is immediate from Theorem 2. Necessity holds because Theorem 5 implies that if $h(S)$ is convex, then $h(S)=S$. Then Theorem 2 implies that $S$ must be quadratically invariant with respect to $G$.

\section{Conclusion}

The main result shows that quadratic invariance is necessary and sufficient for the change of variables $Q=h(K)$ to yield a convex optimization problem. However, this is still only a sufficient condition for tractability.

Indeed, it can happen that $S$ is not quadratically invariant with respect to $G$, yet the set of achievable closedloop maps $f(P, S)=\left\{P_{11}-P_{12} h(K) P_{21} \mid K \in S\right\}$ is affine. A trivial example is the case where $P_{12}=0$ or $P_{21}=0$; here any choice of $S$ works.

Two nontrivial examples are the internally quadratically invariant cases [2], and those derived using variable elimination by Shin et al. [6]. In both of these cases, $h(S)$ is not a convex set, as predicted by Theorem 5 . The general question of characterizing when $f(P, S)$ is convex remains an open problem.

\section{References}

[1] V. D. Blondel and J. N. Tsitsiklis. A survey of computational complexity results in systems and control. Automatica, 36(9):1249-1274, 2000.

[2] L. Lessard and S. Lall. Internal quadratic invariance and decentralized control. In American Control Conference, pages 5596-5601, 2010.

[3] M. Rotkowitz. On information structures, convexity, and linear optimality. In IEEE Conference on Decision and Control, pages 1642-1647, 2008.

[4] M. Rotkowitz and S. Lall. Decentralized control information structures preserved under feedback. In IEEE Conference on Decision and Control, pages 569-575, 2002.

[5] M. Rotkowitz and S. Lall. A characterization of convex problems in decentralized control. IEEE Transactions on Automatic Control, 51(2):274-286, 2006.

[6] H. S. Shin and S. Lall. Optimal decentralized control of linear systems via Groebner bases and variable elimination. In American Control Conference, pages 5608-5613, 2010 .

[7] H. S. Witsenhausen. A counterexample in stochastic optimum control. SIAM Journal on Control, 6:131, 1968. 